\title{
La aparición de los discursos sobre el multiculturalismo en España: el debate del velo en la prensa escrita ${ }^{1}$.
} The emergence of discourse on multiculturalism in Spain: the veil debate in the press.

\author{
Carlos Manuel Abella Vázquez \\ Proxectos. Consultoría e Formación \\ personal@carlosabella.com
}

\begin{abstract}
Resumen
El debate del velo es un ejemplo paradigmático de los complejos debates políticos, mediáticos y sociales que el multiculturalismo trae consigo. En este artículo pretendo analizar los principales argumentos a favor y en contra del uso del hiyab, tanto de modo genérico como en la escuela. Asimismo, examino el debate que tuvo lugar en España en 2002 a raíz del caso de Fátima, una niña marroquí que deseaba acudir a clase con el velo. Para ello, me centro en los editoriales publicados por los tres principales diarios españoles.
\end{abstract}

Palabras clave: multiculturalismo, debate del velo, análisis del discurso, prensa

\begin{abstract}
The veil debate is a paradigmatic example of the complicated political, social and media issue raised by multiculturalism. In this article I analyze the main arguments in favour and against the use of hijab in general, and specifically to its use in school. At the same time, I will examine the Spanish debate on the veil, which took place in 2002, when Fatima, a Moroccan girl, tried to attend school wearing the hijab. I will centre my analysis on editorials published by the three main Spanish newspapers.
\end{abstract}

Keywords: multiculturalism, veil debate, discourse analysis, press

\section{Introducción}

En este artículo pretendo sintetizar los principales puntos de vista existentes en relación con el debate del velo. Asimismo, intento analizar los discursos que los principales diarios españoles han sostenido al respecto en sus editoriales de prensa. En última instancia, se trata de comprobar si el debate del velo es un asunto cerrado sobre sí mismo o, por el contrario, ha de ser entendido como parte de un debate más amplio acerca de la integración de la población extranjera, especialmente la de origen musulmán.

\footnotetext{
${ }^{1}$ Este artículo se basa en varios apartados de mi tesis, titulada Los discursos mediáticos acerca de la inmigración y el multiculturalismo en España: análisis de los editoriales de ABC, El Mundo y El País, 1994-2002 y defendida en septiembre de 2006 en el Departamento de Sociología y Ciencia Política y de la Administración de la Universidade da Coruña.
} 
En el segundo punto de este artículo contextualizo brevemente el actual momento histórico, en el que España se convierte por primera vez en un país receptor de inmigrantes. Asimismo, defino el concepto de debate social como paso previo al análisis del debate del velo.

En el tercer punto examino el debate del velo. Primeramente desde un punto de vista más teórico e histórico. Seguidamente, centrado en el entorno escolar.

En el cuarto punto centro el análisis en las opiniones de la prensa acerca del velo y en el modo en que sus editoriales dieron forma al debate del hiyab en España en febrero de 2002.

En el quinto punto, y a modo de conclusión, se pretende alejar el foco del análisis para contextualizar el debate del velo como parte de una lucha más amplia que caracteriza el actual período histórico.

Los resultados que se exponen forman parte de una investigación llevada a cabo entre 2000 y 2006, para cuya ejecución se analizaron todos los editoriales acerca de la inmigración y el multiculturalismo publicados por ABC, El Mundo y El País entre 1994 y 2002.

\section{La aparición de los debates multiculturales en España}

El surgimiento de los debates sociales relacionados con el multiculturalismo en España está estrechamente unido a la creciente presencia de población extranjera. En este apartado examinaré brevemente, en primer lugar, las distintas fases migratorias que ha atravesado España y, en segundo lugar, el propio concepto de debate social.

\section{Las tres fases migratorias de España}

En el período histórico que comprende desde el último cuarto del siglo XX hasta la actualidad cabe diferenciar tres grandes etapas en relación con los movimientos migratorios internacionales hacia España (Lorenzo Cachón, 2002: pp. 102-108):

1) Hasta 1985.

2) Desde 1986 hasta 1999.

3) A partir de 1999.

Si bien los cambios de fase coinciden con la aprobación de las denominadas Leyes de Extranjería (las Leyes Orgánicas 7/1985, 4/2000 y 8/2000), no es su entrada en vigor la que determina la elección de las fechas, "sino los cambios que se producen en el mercado y otros aspectos institucionales" (Cachón, 2002: 106). Examinemos brevemente cada una de estas fases.

Con anterioridad a 1985, los extranjeros residentes en España eran fundamentalmente europeos, seguidos a bastante distancia por los latinoamericanos y, en tercer lugar, por los norteamericanos. En conjunto, el $90 \%$ de los residentes pertenecían a países de nuestro entorno cultural europeo o latinoamericano. Los primeros provenían de países más desarrollados que España, mientras que los segundos llegaban en muchas ocasiones escapando de las dictaduras existentes en América Latina. Tal y como explica el Colectivo IOÉ, la imagen que los españoles tenían respecto del extranjero era más bien positiva: "aparecía como paradigma de los moderno, de la libertad, el ocio y la opulencia económica" (Colectivo IOÉ, 1999: 168). Esta percepción se irá tornando negativa durante el transcurso de las décadas de los ochenta y de los noventa. 
Durante la segunda fase (1986-1999) se producen cambios importantes y llega una nueva inmigración: "nueva por sus zonas de origen y el nivel de desarrollo de estas zonas (empezando por África - y especialmente Marruecos - y, en la década de los noventa, el este de Europa y países asiáticos); nueva por sus rasgos fenotípicos (...); nueva por las motivaciones económicas de la inmigración y la existencia de un efecto llamada desde el mercado de trabajo español (...); y nueva por ser individual" (Cachón, 2002: 104). Esta inmigración se suma a la ya existente, diversificando así su composición. En esta etapa se inician procesos de reagrupamiento familiar entre los nuevos inmigrantes, aparece una segunda generación, se consolidan las redes migratorias que ligan España con los países de origen, y se constituyen ciertos factores institucionales, como la fijación de cupos anuales. El factor que desencadena esta etapa es, según Cachón, "el «factor atracción», que produce un «efecto llamada» desde la lógica de la reestructuración del mercado de trabajo que se produce en España en esos años y de un cambio notable en el nivel de deseabilidad de los trabajadores autóctonos" (Cachón, 2002: 104-5), cambio que provoca la aparición de determinados nichos laborales para la población extranjera menos cualificada.

El año 2000 marca la entrada en la tercera fase migratoria. Se alcanza por vez primera el millón de residentes extranjeros, aunque lo característico no es tanto este volumen (todavía modesto, sobre todo en comparación con los países europeos) como la continua ampliación y diversificación de la inmigración. Distintas razones explican el inicio de esta etapa. En lo relativo al mercado laboral, "a los sectores de actividad/ocupaciones/comarcas donde habían aparecido los «nichos laborales» que han ido ocupando progresivamente los inmigrantes desde mediados de los ochenta, y donde su presencia se ha consolidado, han venido a añadirse nuevas ramas/ocupaciones/comarcas que demandan inmigrantes por falta de trabajadores españoles dispuestos a trabajar en ellas". Lo cual ha provocado el comienzo de una "re-etnoestratificación en la fuerza de trabajo inmigrante" o, dicho de otro modo, "desplazamientos de unos colectivo por otros" (Cachón, 2002: 106). Al mismo tiempo, las redes migratorias se consolidan, lo que posibilita e impulsa los procesos de reagrupamiento familiar y la aparición de una cada vez más numerosa segunda generación de inmigrantes.

En esta última etapa surgen, por tanto, cuestiones relacionadas con la ciudadanía, y que tienen que ver con la enculturación y asentamiento de la población extranjera en un territorio que construyen tanto física como socialmente. Se consolidan también en esta etapa instituciones no estatales (como los sindicatos) que buscan la integración social de los inmigrantes, surgen conflictos y luchas reivindicativas con el fin de mejorar las condiciones vitales y laborales, y aparece "en el horizonte también la cuestión de la participación política" (Cachón, 2002: 107). En definitiva, en esta tercera etapa comienzan a cobrar importancia cuestiones relacionadas con la ciudadanía y el multiculturalismo, y la inmigración pasa a ser un asunto de relevancia mediática, política y social. Es precisamente en este contexto en el que el debate del velo ha tenido lugar, como veremos más adelante.

\section{El concepto de debate social}

De una forma global, es posible afirmar que a partir de la década de 1990 España fue testigo de un amplio debate social en relación con la inmigración y el multiculturalismo. Entre las características definitorias de este hipergénero comunicativo que señala Antonio Bañón (2002: 23-29), me gustaría resaltar aquí las siguientes: 
En primer lugar, un debate social supone "la aparición pública de opiniones dispares a propósito de algún tema" (Bañón, 2002: 24), a lo largo de un período, cuyo interés es compartido por amplios sectores sociales.

En segundo lugar, se produce la participación de todos aquellos actores sociales, individuales o colectivos, que así lo desean: políticos, medios de comunicación, diversas ONG, etc. No obstante, ni todos los foros ni todos los actores tienen la misma capacidad de hacerse escuchar. Además, "la pluralidad de medios y discursos (...) no implica la pluralidad de versiones" (Bañón, 2002: 25).

Tercero, los contenidos adquieren diversos tipos discursivos (entrevistas, editoriales, pero también encierros, conferencias, etc.). En este caso, he decidido centrarme en los editoriales como tipo discursivo que refleja explícitamente el posicionamiento ideológico de cada diario y contribuye decididamente a la generación de la opinión pública, al tiempo que influye en (y es influido por) los discursos de los partidos políticos.

En cuarto lugar, es común que se generen debates sectoriales: inmigración y legislación, inmigración y vivienda, inmigración y educación, inmigración e integración, etc. Los debates sectoriales, tal y como los define Bañón, tienen que ver, a efectos analíticos, con las distintas categorías temáticas (subjects) en el sentido que les da Teun A. Van Dijk (véase, por ejemplo, Van Dijk, 1985).

Una quinta consideración: el debate social está compuesto por "discursos de índole intragrupal (incluido el discurso intragrupal acerca de otros) e intergrupal" (Bañón, 2002: 26). Como veremos, el análisis posterior se centra en, y parece dejar clara la existencia de, distintos discursos que desde la mayoría social (representada por la prensa escrita) se sostienen acerca de las minorías, en este caso acerca de las que proceden de los movimientos migratorios, y fundamentalmente de países musulmanes.

Por último, el debate social confluye en los medios de comunicación masiva (prensa de referencia), que sirven de vehículo de articulación del mismo. En efecto, en este caso podemos considerar que $A B C$, El Mundo y El País constituyen la prensa de referencia española durante el período considerado.

En definitiva, si tenemos en cuenta los criterios anteriores, se puede afirmar con rotundidad que fundamentalmente a partir de la segunda mitad de la década de 1990, España ha sido testigo de un amplio debate sobre la inmigración y el multiculturalismo, el cual ha ido ganando intensidad en los últimos años. De forma más específica, se puede aseverar que a principios de 2002 se produjo un debate en torno al uso del velo en la escuela, como tendremos ocasión de comprobar más adelante.

\section{El debate del velo: aproximación histórica y teórica}

El debate acerca del velo no se deja encasillar fácilmente en los habituales esquemas ideológicos de las democracias occidentales. En efecto, cruza las habituales distinciones políticas y crea extraños compañeros de viaje: hay posiciones a favor y en contra en la izquierda y dentro del movimiento feminista. Dentro del propio colectivo de musulmanes se pueden identificar posiciones favorables al uso del pañuelo islámico que esgrimen argumentos diferentes e incluso irreconciliables, pero incluso también posturas críticas para con el uso del velo (sobre todo, con su instrumentalización proselitista). Desde los sectores conservadores de las sociedades de recepción las posiciones suelen coincidir en torno a la idea de la prohibición o, al menos, el repudio del hiyab. 
Antes de comenzar a exponer los distintos puntos de vista teóricos y de analizar los discursos mediáticos, es imprescindible realizar una aclaración terminológica y conceptual. En ocasiones se emplean indistintamente términos como chador, velo o hiyab, como he podido constatar en los editoriales de prensa. Djaouida Moualhi realiza una pequeña síntesis del significado de los distintos términos que resulta muy clarificadora. Esta autora describe, en primer lugar, las prendas tradicionales, como el hayek y la melaya o jelaba. El hayek es "una típica capa de seda, generalmente de color blanco o beige y sin ninguna costura, que solían llevar tradicionalmente las mujeres magrebíes. La melaya o jelaba es tradicionalmente de tela gruesa, dependiendo su color de las regiones. A diferencia del hayek, esta prenda es cosida, con mangas, y puede llevar capucha" (Moualhi, 2000: 298, nota a pie $n^{0} 10$ ). Estas prendas habrían sido sustituidas recientemente por el hiyab y por el chador, que no significan lo mismo y, de hecho, son muy diferentes entre sí: "El hijab es un pañuelo que cubre la cabeza y puede ser de diferentes colores, llevarse con cualquier vestido, falda o pantalón amplio que no muestre las formas, y se suele llevar en el Magreb y países de Oriente. En cambio, el chador es una prenda única cosida y que cubre todo el cuerpo incluyendo la cabeza y debe ser de color negro, típica de Irán y ajena a las tradiciones norteafricanas" (Moualhi, 2000: 298, nota a pie $\mathrm{n}^{\circ} 10$ ). Así pues, se pueden emplear indistintamente los términos velo, hiyab, e incluso pañuelo, pero el chador es una prenda diferente.

A efectos analíticos, cabe diferenciar dos grandes grupos de cuestiones teóricas y prácticas dentro del debate general acerca del velo. En primer lugar, uno más general que tiene que ver con los significados y las interpretaciones del pañuelo islámico en los países receptores de población inmigrante procedente de países musulmanes. En segundo lugar, otro más específico que alude al uso del hiyab en las escuelas públicas de dichos países y tiene que ver fundamentalmente con la relación entre laicismo y religión. Quienes se oponen al velo suelen argumentar que es un símbolo religioso de sumisión de las mujeres a los varones y que, por tanto, su uso no debe ser permitido en los colegios públicos, pues atenta tanto contra la neutralidad religiosa de los centros, como supone una inaceptable quiebra de la igualdad entre los sexos. En las páginas que siguen intentaremos adentrarnos en este debate poniendo de relieve los principales argumentos esgrimidos desde unos y otros sectores.

\section{El debate del velo en las sociedades occidentales}

De un modo general, Lena de Botton, Lidia Puigvert y Fátima Taleb parten del cuestionamiento de "que el hiyab deba ser interpretado necesariamente como el resultado de una imposición masculina y patriarcal" (Botton, Puigvert y Taleb, 2004: 13). La hipótesis de estas autoras es que el hiyab "debe ser contemplado como un síntoma más de todo el camino que aún nos queda por avanzar a las mujeres en el control de nuestra propia vida" (Botton, Puigvert y Taleb, 2004: 13.). Así pues, tanto la obligación como la prohibición de llevarlo serían formas de atentar contra la libertad de elección de las mujeres. La proscripción de ponerse el velo es algo que puede ocurrir en las sociedades occidentales partiendo de dos tipos distintos de actitudes. En primer lugar, culpabilizadoras: se cuestiona el derecho a elegir si llevar o no hiyab en nombre del respeto a la libertad religiosa de toda la sociedad. En segundo lugar, paternalistas: se les prohíbe portar el velo como medio para superar su opresión.

En todo caso, lo realmente importante para estas autoras es que ambos tipos de conductas, prohibición u obligatoriedad de portar el velo, forman parte "de patrones jerárquicos masculinos y patriarcales, donde la mujer ocupa una posición inferior en cuanto a sus derechos a decidir y al reconocimiento de las capacidades necesarias para ello" (Botton, Puigvert y Taleb, 2004: 14). El 
núcleo central de su argumentación se sitúa, así, en la necesidad de asegurar los medios para que se dé una efectiva libertad de elección a las mujeres, a todas las mujeres. En este sentido afirman que la prohibición del velo "está perjudicando gravemente no sólo la libertad de las mujeres musulmanas de escoger libremente cómo desean vestirse, sino la libertad de toda mujer a expresarse como desee" (Botton, Puigvert y Taleb, 2004: 40). Lo que está en juego para estas autoras, pues, es una sociedad más igualitaria.

En este sentido, resulta importante la constatación empírica de que "muchas mujeres musulmanas deciden por sí mismas llevarlo de forma libremente escogida. La decisión de ponerse el hiyab no implica pasividad ni opresión cuando es el fruto de una reflexión madurada en vez de una imposición" (Botton, Puigvert y Taleb, 2004: 21). De esta forma, muchas mujeres musulmanas que llevan hiyab en las sociedades europeas están afirmando "un tipo de feminidad y [una] identidad cultural diferente a la hegemónica"².

Así pues, el uso del hiyab, pero también de otras prendas como los pantalones, los sujetadores, las minifaldas o los bikinis "debe ser analizado en atención al respeto a la decisión de las mujeres, de lo que dependen sus consecuencias transformadoras y emancipadoras, así como sus efectos superadores de la desigualdad que les impide alzar su voz" (Botton, Puigvert y Taleb, 2004: 30).

En este sentido, podemos pensar que el uso de una determinada prenda (como las minifaldas) degrada objetivamente a las mujeres y las convierte en objetos de consumo masculino, todo ello bajo la ilusión de una aparente emancipación; se interpretaría, de esta forma, que estarían alienadas. Alternativamente, se puede interpretar que su uso supone la liberación de unos patrones culturales y religiosos conservadores en los que la sumisión de las mujeres y su sujeción a los roles tradicionales son elementos básicos. Ambas interpretaciones pueden ser igualmente válidas o incorrectas, pues pecan de un fallo común: no cuentan con el sentido que dan a sus acciones las propias protagonistas. La sola aplicación de una perspectiva etic en casos como este puede producir interpretaciones alejadas de la realidad y de los verdaderos sentidos que los actores sociales dan a sus actos. Cuando estas interpretaciones se realizan sobre prácticas de otras culturas, el riesgo de malinterpretar y de caer en prejuicios etnocéntricos es mayor.

Este es, precisamente, el tipo de crítica que algunas feministas musulmanas hacen de aquellas feministas occidentales que presentan a las mujeres de sus sociedades "como modelos para todas las mujeres del mundo y [se niegan] a considerar que existen otros itinerarios diferentes al suyo para modificar las relaciones entre sexos" (Sophie Bessis, 2002: 308, nota a pie $n^{\circ}$ 40). En esta impugnación, por otra parte, es frecuente que estas mujeres del Tercer Mundo que desean combatir el patriarcado se vean influidas por los discursos nacionalistas e identitarios (profundamente conservadores, por tanto) promovidos por los movimientos islamistas de sus países.

${ }^{2}$ Botton, Puigvert y Taleb, 2004: 21. Diversos estudios sugieren que las mujeres y las chicas musulmanas deciden llevar el velo por una gran multiplicidad de razones. Véanse, por ejemplo, Chouki El Hamel, 2002, especialmente pp. 297-301, o Jen'nan Ghazal Read y John P. Bartkowski, 2000: 401 y siguientes. En este último artículo se muestran los resultados de un estudio de campo en Austin (Texas) para indagar las razones por las que algunas mujeres musulmanas deciden ponerse el velo y otras no. En esta investigación se descubrió una clara divergencia entre los discursos de las elites religiosas acerca del hiyab y el de las propias mujeres y que, en todo caso, demostraban ser individuos activos y no sujetos pasivos: "both the veiled and unveiled women in our study clearly exercise agency in crafting their gender identities" (Read y Bartkowski, 2000: 411). 
Sin embargo, hay algo de cierto en ambos puntos de vista. En muchas ocasiones, el uso del hiyab es efectivamente impuesto y, de este modo, se convierte en un símbolo de la sumisión de las mujeres a los varones. Esta imposición puede ser, en cierto modo, aceptada. Como tal aceptación impuesta puede ser una estrategia de las mujeres musulmanas para no perder espacio público. De un modo ciertamente paradójico, "negocian su emergencia social adoptando lo que fue siempre el más espectacular símbolo de alienación" (Bessis, 2002: 188). En este sentido, ponerse el hiyab es para esta autora una concesión vestimentaria.

Queda claro que en estos casos, más frecuentes en los países del Magreb que en los europeos (pero que, desde luego, también se da en estos últimos, como se describe en Fadela Amara y Sylvia Zappi, 2004), el uso del velo no se debe a una elección verdaderamente libre y autónoma, sino que es un modo de ceder ante las enormes presiones sociales para, a cambio, conseguir ciertas cotas de participación en la esfera pública que, en caso contrario, estarían vedadas. Así pues, en estos casos ponerse el hiyab es una forma de escapar a la asfixiante presión del entorno, al sentimiento de estar permanentemente vigiladas, bajo sospecha: "Es tan fuerte el peso del modelo dominante reforzado por la propaganda islamista, tan intensa la culpabilización de las mujeres, tan mal vivida la hibridación de su estatuto, tan doloroso el sentimiento de estar desprovista de identidad, que ven en el hiyab el único medio de reconciliarse con ellas mismas, de afirmar al llevarlo su verdadera naturaleza árabemusulmana, de corresponder a lo que se espera de ellas, y seguir siendo libres para educarse, trabajar y poder elegir un consorte" (Bessis, 2002: 188).

Por otra parte, esta utilización del hiyab por parte de los movimientos islamistas norteafricanos es muy reciente, pues data de las décadas de 1980-90, y se enmarca en un proceso social de retroceso que viene a quebrar la relativa emancipación que habían logrado las mujeres del Magreb durante los años 60 del siglo XX. El crecimiento de las redes de apoyo fundamentalistas, que en ocasiones llegan a constituir auténticas administraciones paralelas al Estado, con su ejercicio de proselitismo prohiyab, ayuda a explicar la gran extensión (y aceptación, impuesta o no) que hoy en día llega a tener el uso del velo en los países norteafricanos (Sophie Bessis y Souhayr Belhassen, 1994).

Esta estrategia de aceptación del hiyab para no renunciar a los espacios públicos es a la vez señal de que velo e islamismo no son sinónimos, por un lado, y del poder que han alcanzado los movimientos fundamentalistas para que muchas mujeres prefieran ceder y ponerse el velo. Como dicen Bessis y Belhasen, el hecho de que el hiyab deje de ser "un signo de unión y bandera de una ideología" de corte islamista y se produzca la "banalización" de su uso, es la "prueba tangible el éxito del proselitismo islamista" (Bessis y Belhassen, 1994: 185).

Dentro de los países receptores de población musulmana, cabe interpretar el debate sobre el hiyab como el centro de una batalla entre los sectores más fundamentalistas del Islam y determinados sectores de la sociedad de recepción. Analizado desde fuera, adquiere la forma de una pugna entre varones musulmanes, que representan a los sectores religiosos más extremistas, y algunos varones de las sociedades occidentales, que representan a las distintas elites sociales. Las mujeres se convierten en una especie de objeto transaccional (Rosa Cobo, 1999) de esta disputa: son objeto de un debate en el que no se les deja participar en tanto sujetos activos, a pesar de ser las principales afectadas. Como dice Cobo: "en las discusiones sobre multiculturalismo siempre aparecen algunas prácticas culturales, cuyas destinatarias son las mujeres (mutilación genital femenina o chador), como el ejemplo pertinente que justifica una u otra posición. Las mujeres no son pensadas como sujetos de derechos sino como objetos transaccionales de las discusiones de los varones expertos en estos debates" (Cobo, 1999: 59). 
Pero este planteamiento es también habitual en los países de origen de las mujeres musulmanas. Como afirma Bessis, la conservación de los roles tradicionales de las mujeres es considerada como algo fundamental para la reproducción y el mantenimiento de la identidad colectiva. Forma parte de la reacción contra el imperialismo occidental y su manifestada superioridad, su descarado y arrogante etnocentrismo. Es, así, una estrategia política de los sectores conservadores del mundo árabe y musulmán en su proyecto de lucha contra la modernidad occidental y de restauración (invención) de una supuesta identidad pasada. El feminismo es demonizado al ser presentado como "una ideología puramente occidental, antimasculina y causante de los mayores desarreglos sociales" (Bessis, 2002: 308). La nación y la religión son los dos principales sectores de esta regeneración reactiva y las mujeres, como tantas veces en la Historia, las principales depositarias de las esencias nacionales, culturales y religiosas. En efecto, la mitificación nacionalista de las mujeres es algo que trasciende los contextos culturales y religiosos y, por tanto, no es atribuible en exclusiva a los movimientos islamistas actuales. Esta mitificación hace de las mujeres las matrices biológicas y las transmisoras de las esencias culturales de la nación: "Women are not only the biological reproducers of an ethnic group, but also the 'cultural carriers' who have the key role in passing on the language and cultural symbols to the young. In nationalist discourses, women often serve as the symbolic embodiment of the national identity and distinctiveness. They nurture and support the warrior-citizens (invariably seen as male)" (Stephen Castles y Alastair Davidson, 2000: 121).

Vemos así que los movimientos feministas del Tercer Mundo deben luchar contra los sectores fundamentalistas de sus países, sin duda su principal enemigo, para reivindicar unas nuevas relaciones de género basadas en la igualdad entre varones y mujeres. Pero también deben enfrentarse a algunos sectores del feminismo occidental para reivindicar el valor de algunas de sus prácticas culturales y demandar el reconocimiento a sus especificidades como mujeres musulmanas: a su identidad en tanto mujeres y musulmanas.

A todo este entramado de posiciones políticas e ideológicas hay que añadir también un movimiento feminista secular en los países musulmanes que, como afirma El Hamel (2000: 302), conecta el velo con la reclusión de las mujeres, la cual asegura el rol de los varones como proveedores y guardianes de las mujeres. Una de sus máximas representantes, Nawal El Saadawi, se posiciona claramente en contra del velo en los siguientes términos: "The call to liberate the mind, or to raise the veil from the mind (...) is an essential for the liberation of the Arab person, man or woman, but especially woman. For she is ruled by two authorities (inside and outside home) which deprive her of her rights over her own mind and body and from becoming the moving force behind her own deeds" (El Saadawi, citado en El Hamel, 2000: 302).

Lo que he expuesto hasta aquí permite apreciar la pluralidad de interpretaciones y motivaciones que se pueden esconder detrás del velo. Las siguientes tipologías pueden ayudarnos a sintetizar lo expuesto y a clarificar el debate.

Según Moualhi, actualmente muchas de las mujeres que portan el velo en los países europeos lo hacen movidas, en primer lugar, por una convicción personal de índole ética, como la expresión voluntaria de su fe y sumisión a Dios (que no a los varones).

En segundo lugar, algunas jóvenes de origen magrebí pueden usarlo "como reivindicación de una identidad diferenciada" (Moualhi, 2000: 298.), esto es, como un elemento de distinción cultural y religiosa de tipo comunitario. 
En tercer lugar, la autora también reconoce que "cediendo a las presiones sociales del entorno, otras lo llevan para no ser prejuzgadas ni ser molestadas en los lugares públicos, y en otros casos por imposición familiar" (Moualhi, 2000: 298). En estos casos, pues se trata de un sistema de discriminación y falta de libertad.

Esto es precisamente lo que denuncia Fadela Amara cuando describe la situación de muchas chicas musulmanas en algunas barriadas obreras francesas. Desde su punto de vista, muchas ceden a las sofocantes presiones masculinas y en este sentido el velo es una especie de armadura contra las agresiones de los chicos, algo que hace que sean respetadas (Amara, 2004). El hecho de que muchos varones musulmanes hayan hecho del velo un elemento de su lucha contra la sociedad francesa y de símbolo de su poder sobre sus mujeres es lo que lleva a esta autora a estar decididamente en contra del hiyab.

Por otra parte, el informe Stasi (que será tratado en mayor profundidad en el siguiente punto) se hace también eco de la situación que describe Amara: "Les jeunes femmes se retrouvent victimes d'une résurgence du sexisme qui se traduit par diverses pressions et par des violences verbales, psychologiques ou physiques. Des jeunes gens leur imposent de porter des tenues couvrantes et asexuées, de baisser le regard à la vue d'un homme; à défaut de s'y conformer, elles sont stigmatisées comme «putes». (...) Dans ce contexte, des jeunes filles ou des femmes portent volontairement le voile, mais d'autres le revêtent sous la contrainte ou la pression. (...) Les jeunes filles, une fois voilées, peuvent traverser les cages d'escalier d'immeubles collectifs et aller sur la voie publique sans craindre d'être conspuées, voire maltraitées, comme elles l'étaient auparavant, tête nue. Le voile leur offre ainsi, paradoxalement, la protection que devrait garantir la République “3 ${ }^{3}$ (Commission de réflexion sur l'application du principe de laïcité dans le République, 2003: 47).

En cuarto lugar, por último, hay mujeres musulmanas que desean oponerse a las otras (las occidentales no veladas), como en el caso de algunas islamistas. En este caso, el velo adquiere una "connotación política" (Moualhi, 2000: 299) que marca la pertenencia a una cultura islámica y sirve de elemento aglutinador.

Botton, Puigvert y Taleb proponen otra tipología, basada en la distinción entre velo personal y velo político. El primero "nace desde la libertad de la mujer de escoger por diferentes motivos, religiosos, éticos, intelectuales o culturales, entre otros. En cualquiera de estos casos, el velo personal se manifiesta como una libertad de opción" (Botton, Puigvert y Taleb, 2004: 34-5).

Por su parte, el velo político "es aquél que utiliza a las mujeres como instrumento de control político, silenciándolas y excluyéndolas para recluirlas en una posición de desventaja dentro un espacio social y político dado" (Botton et al, 2004: 34). En este sentido, no está de más recordar que el primer uso político del velo data del proceso de colonización de Argelia, durante el cual los franceses llevaron a

\footnotetext{
${ }^{3}$ Las jóvenes son victimas de un resurgimiento del sexismo que se traduce en diversas formas de presión y violencia verbal, psicológica o física. Los jóvenes les imponen formas de vestir que cubren lo más posible y son asexuadas y las obligan a bajar la mirada en presencia de un hombre: si se niegan a obedecer, son estigmatizadas como "prostitutas" (...). En este contexto, aunque hay niñas o mujeres jóvenes que sí usan voluntariamente el velo otras que lo hacen por presión o miedo. (...) Una vez que usan el velo pueden atravesar las escaleras del portal e ir por la vía publica sin miedo a ser insultadas o maltratadas como les ocurría si no lo llevaban. El velo les ofrece, de esta forma la protección que debía garantizarles la República
} 
cabo una campaña de glorificación de las liberadas mujeres bereberes, que no llevaban velo. Ello hizo que, posteriormente, el hiyab se convirtiera en un elemento aglutinador en la lucha por la independencia (Moualhi, 2000). En la actualidad, este uso político se da en Afganistán, pero también en Europa cuando se prohíbe su uso: "El hiyab, también aquí, se utiliza como arma arrojadiza, justamente por su omisión de la dinámica social, porque es convertido en motivo de exclusión" (Botton et al, 2004: 34).

Desde luego, es necesario tener en cuenta este uso del hiyab que se hace desde determinados sectores de las sociedades receptoras. El velo es un elemento más de su ofensiva contra el Islam. Como afirma Moualhi, en muchos discursos subyace una visión homogeneizante de esta religión. Basados en estereotipos etnocéntricos, toman la parte por el todo y obvian "la diversidad sociocultural, ético-religiosa y política existente en y entre los países árabes y/o musulmanes" (Moualhi, 2000: 302). Y aquí desempeña su papel el velo: se usan los estereotipos sobre la situación de las mujeres árabo-musulmanas como el "instrumento más eficaz para demonizar sus sociedades" (Moualhi, 2000: 303).

Algunos de los argumentos esbozados se trasladan al debate del velo en la escuela, si bien aquí entra en liza una variable importante: el laicismo del sistema educativo.

\section{El velo en la escuela: ¿un debate acerca de la laicidad de la enseñanza?}

El debate acerca de la prohibición o permisión del hiyab en las escuelas públicas encendió vivas polémicas en Francia en 1989 y en los años siguientes. El origen del denominado affaire des foulards se encuentra en el documento redactado por el consejo escolar del instituto de enseñanza media de Creil, una pequeña localidad situada al norte de París, que incluía una cláusula de laïcité en la que se exigía "discreción para portar distintivos de carácter religioso" (Danièle Hervieu-Léger, 1999: 113). En la práctica, esto se tradujo en la prohibición de entrar en las aulas a aquellas alumnas que portaran el hiyab y la expulsión de las que no acataran la norma, lo que provocó protestas de algunas niñas y sus familias. Tras algunas negociaciones, padres y consejo escolar llegaron a un acuerdo, pero el asunto del velo ya había trascendido los límites locales para saltar a la prensa nacional. Para entonces ya se había convertido en un debate caracterizado por la excesiva dramatización y la radicalización ideológico-política, proceso en el que la prensa desempeñó un papel clave ${ }^{4}$.

Podríamos sintetizar las preguntas clave de la discusión que tuvo lugar entonces como sigue: ¿Se debe permitir que las niñas asistan con la prenda tradicional islámica a las escuelas públicas, laicas? ¿O se debe respetar el derecho a la libertad religiosa, expresado en el atuendo? ¿Es incompatible llevar el velo con el respeto al laicismo? ¿No supone la exhibición del hiyab una inferiorización de las niñas? ¿O se trata de una libre elección individual, una manifestación voluntaria de la propia identidad cultural? ¿Supone la prohibición de esta prenda tradicional una muestra de islamofobia por parte del supuestamente liberal Estado francés? ¿O es solamente una demostración del carácter laico y aconfesional del Estado? Si es así, ¿por qué la exhibición de símbolos religiosos ostensibles de otras confesiones (católica, judía) no fue censurada al mismo tiempo?

\footnotetext{
${ }^{4}$ Para el análisis de la cronología de los acontecimientos, desde el citado documento del instituto hasta la generación de un apasionado debate social, político y mediático acerca del asunto del velo en Francia, véanse por ejemplo Hervieu-Léger, 1999: 113-121; Caitlin Killian, 2003: 567-573; Jane Freedman, 2004: 12-16.
} 
Digo "al mismo tiempo" porque no fue hasta marzo de 2004 que se prohibió la exhibición de símbolos religiosos ostensibles de cualquier confesión en las escuelas públicas francesas, tanto del hiyab como de los crucifijos de grandes dimensiones o la de la tradicional kippa judía (Loi $n^{\circ}$ 2004-228). Esta ley se aprobó siguiendo las recomendaciones de la Comisión Stasi, creada por el presidente Jacques Chirac para examinar la cuestión del laicismo en Francia. A pesar de proscribir símbolos religiosos diversos, es inevitable analizar esta ley como consecuencia del asunto del velo que tuvo su origen en 1989.

Desde un punto de vista histórico, el laicismo es un valor central de la identidad nacional francesa, fuertemente enraizado con el universalismo republicano derivado de la Revolución de 1789. Uno de los valores centrales del republicanismo francés es "the disappearance of differences through the assimilation of all to one legitimate culture" (Jane Freedman, 2004: 10). En este sentido, el secularismo trata de lograr la igualdad en la esfera pública en relación con la religión "by removing all religious observance from public institutions so that the private religious divides that exist in society are overcome" (Freedman, 2004: 10).

Inicialmente, el laicismo trata de implantarse para limitar las influencias del catolicismo en la esfera pública, algo que se consigue con la Ley de 1905 que consagra la separación de las iglesias y el estado. El Informe de la Comisión Stasi resume así los logros de esta Ley: "La dissociation de la citoyenneté et de l'appartenance religieuse est affirmée; la religion perd sa fonction d'instance de socialisation officielle; enfin la France cesse de se définir comme nation catholique tout en renonçant au projet d'une religion civile républicaine" ${ }^{\prime 5}$ (Commission de réflexion sur l'application du principe de laïcité dans le République, 2003: 11). En este proyecto, por tanto, la escuela ocupa un lugar central, pues debería proporcionar una educación igual a todos los niños y niñas que trascendiera los distintos orígenes sociales y culturales de los mismos. La escuela es así un lugar de creación de futuros ciudadanos que han de compartir una cultura cívica común.

¿Supone el uso del velo una contravención de estos principios? El Informe de la Comisión Stasi es claro a este respecto. El hiyab no sólo es contrario al laicismo, sino que además se interpreta como el resultado de un ejercicio de poder sobre las chicas musulmanas, que sufrirían así una presión intolerable y serían las víctimas silenciosas de los grupos islamistas. Por tanto, se considera que la República debe intervenir para proteger a estas chicas y asegurar que la escuela sea para ellas un lugar de libertad y emancipación: "L'espace scolaire doit rester pour elles un lieu de liberté et d'émancipation" (Commission de réflexion..., 2003: 58).

Por tanto, esta comisión propone incluir la siguiente disposición en una ley sobre laicismo: "Dans le respect de la liberté de conscience et du caractère propre des établissements privés sous contrat, sont interdits dans les écoles, collèges et lycées les tenues et signes manifestant une appartenance religieuse ou politique. Toute sanction est proportionnée et prise après que l'élève a été invité à se conformer à ses obligations" ${ }^{\prime 6}$ (Commission de réflexion..., 2003: 58). El informe especifica,

\footnotetext{
${ }^{5}$ La religión pierde su función de instancia oficial de socialización; al fin Francia deja de definirse como una nación católica renunciando al tiempo al proyecto de una religión civil republicana.

${ }^{6}$ En respeto de la libertad de conciencia y de carácter propio de los establecimientos privados bajo contrato con el estado, se prohíben en las escuelas, colegios y liceos las vestimentas y signos que manifiesten una pertenencia religiosa y política. Toda sanción será proporcional y aplicada después de que el alumno haya sido invitado a cumplir sus obligaciones.
} 
seguidamente, que entran bajo esta rúbrica signos religiosos ostensibles como la kippa, una gran cruz o el velo, pero no signos discretos como medallas, pequeñas cruces, estrellas de David, manos de Fátima o ejemplares pequeños del Corán. Estas recomendaciones fueron finalmente tenidas en cuenta en la Loi $n^{\circ}$ 2004-228, con lo que se ponía fin, legislativamente hablando, al debate iniciado en 1989.

Desde un punto de vista alternativo, algunos han argumentado que resulta contradictorio imponer por la fuerza los valores republicanos. Como afirma Ignasi Álvarez, "los muy estimables valores laicos, republicanos y de igualdad de las mujeres no deben imponerse por la fuerza; (...) si la escuela difunde esos valores, no parece muy sabio prohibir que esas niñas accedan a ella" (Álvarez Dorronsoro: 1994: 50-51). En este sentido, algunos consideraron que, si bien el hiyab podía no ser compatible con el laicismo, no debería ser en ningún modo un elemento que evitara la escolarización de las niñas, y que el valor integrador de la escuela debía prevalecer sobre el laicismo: prohibir que las niñas asistieran a clase sería más excluyente que permitir que portaran el velo. Esta fue, de hecho, la postura del ministro de Educación francés Lionel Jospin durante el asunto del velo en 1989 (Killian, 2003: 571)

También puede resultar paradójico que se argumente que llevar el hiyab es una evidencia de la falta de libertad "cuando en las sociedades occidentales la presión social es más bien en contra del velo. En sociedades donde se proclama la libertad de culto en el vestir, esa prenda no tendría que ser vista más que como un signo inocente del ejercicio de esos derechos, comparable al corriente colgante en forma de crucifijo" (Moualhi, 2000: 299). En este sentido, algunos autores consideran que resulta curioso que exista tanta preocupación por si las niñas musulmanas son obligadas a portar el velo o lo llevan libremente y no exista una paralela preocupación por la libertad de las niñas cristianas para llevar o no crucifijos u otro tipo de distintivos religiosos. Tal vez subyazca a ello un punto de vista etnocentrista que ve en el crucifijo un símbolo respetable (e inocente), desprovisto de connotaciones que resultarían obvias para personas de otras religiones o culturas. Como dice El Hamel al exponer los resultados de su investigación: "Pro-hijab Muslims in France acknowledge that the Muslim children do not choose the hijab, but they argue whether the Christian children choose to wear a long skirt rather than pants, and go to church every Sunday. (....) Obviously the culture of the religions is not chosen. In sum, the Muslims I interviewed wondered why the Muslim children in France are expected to choose their cultural and religious symbols for their identity" (El Hamel, 2000: 303). Sobre todo si se tiene en cuenta que, además, la gran mayoría de los niños y niñas musulmanes no se visten conforme a los códigos tradicionales islámicos.

Para Álvarez Dorronsoro, el fondo del debate no es el carácter laico o religioso de la escuela pública, ni el papel de las mujeres en las sociedades contemporáneas, sino una cuestión de ausencia de reconocimiento y respeto a otras culturas: "Lo anterior muestra que algunos conflictos interculturales emblemáticos, como el de la supuesta incompatibilidad de las creencias islámicas con los valores republicanos, o el del chador, no nacen de una radical incompatibilidad de valores entre las culturas, sino de la existencia de un alto nivel de intolerancia en una parte de la sociedad receptora respecto a los comportamientos de algunos colectivos inmigrantes" (Álvarez Dorronsoro: 1994: 51).

En este sentido, el rechazo del velo debe ser interpretado dentro de una concepción más amplia acerca de la integración de los inmigrantes en las sociedades receptoras y, concretamente, como parte de la estrategia asimilacionista y del rechazo de las políticas multiculturales o del derecho a la diferencia. Esta es la argumentación que sostiene, por ejemplo, Freedman (2004). 


\section{El debate acerca del velo en la prensa escrita.}

El multiculturalismo ha dado pie a la aparición de multitud de debates sociales. En última instancia, la cuestión básica que subyace a todos ellos es si la aplicación de políticas multiculturales favorece o entorpece la integración de los inmigrantes extranjeros, si aumenta la unidad social y el compromiso de la población inmigrada con la sociedad de recepción o, por el contrario, supone la fragmentación y la balcanización social. El debate acerca del velo en la escuela es uno de los campos en los que se libra esta batalla entre partidarios y detractores del multiculturalismo. En mi opinión, el asunto del hiyab alcanza la relevancia mediática, social y política que tiene porque en él se cruzan tres ejes fundamentales: se trata de una práctica religioso-cultural ajena, de procedencia árabomusulmana, y que afecta a las mujeres.

En efecto, en mi investigación he detectado que, a pesar de que las diferencias culturales son introducidas en España por muy distintos colectivos nacionales o culturales, la prensa muestra una cierta predisposición a centrarse en las costumbres de origen musulmán, y éstas parecen ser las únicas que se convierten en objeto de debate: sea la enseñanza del Islam, el velo en la escuela, la mutilación genital femenina o el matrimonio pactado entre padres. Además, un elemento común subyace a casi todos estos temas: las mujeres como objeto de opinión. En efecto, la prensa suele recurrir al género como paradigma de la contraposición entre el mundo occidental y el islámico. De un modo u otro, las mujeres pasan a ser parte de la cultura. En cierta forma, el debate sobre las mujeres y / en el Islam pasa a ser un debate entre varones de uno y otro lado, opinando acerca de sus respectivas mujeres (o de las del otro).

En España, el debate del velo tuvo lugar a principios de 2002 (casi trece años después, por tanto, de que comenzara en Francia) y el detonante de su aparición fue el deseo de Fátima, una niña marroquí de 13 años, de acudir a clase con el hiyab, y la subsiguiente negativa del colegio privado concertado católico al que asistía a que lo hiciera. Además de la multitud de noticias y artículos de opinión publicados, los tres principales diarios españoles escribieron editoriales íntegramente dedicados a este asunto. En concreto, los editoriales que analizaré tienen fecha de 16 de febrero (El Mundo y ABC), 17 (El País) y 18 (El Mundo). A no ser que se especifique lo contrario, por tanto, las citas que aparecerán a continuación se referirán a estos días. Por otra parte, además de estos editoriales dedicados únicamente a opinar acerca del asunto del velo, los tres diarios manifestaron sus puntos de vista al respecto en otros editoriales dedicados de forma más genérica a la inmigración o al Islam.

\section{Puntos de partida de $A B C$, El Mundo y El País}

Antes de presentar las posturas básicas de cada diario, resulta importante recordar la matización conceptual introducida en el punto 3.1, pues no es lo mismo valorar el uso del pañuelo que cubre sólo la cabeza (hiyab) que la tradicional vestimenta que cubre todo el cuerpo de la mujer (chador). En este caso, la niña (o el padre de ésta) deseaba poder acudir a la escuela con el hiyab, esto es, con el pañuelo que cubre el pelo.

Esta aclaración resulta importante porque, a pesar de ello, $A B C$ contextualiza su opinión afirmando que el colegio al que la niña asiste se niega "a aceptar el uso de esa prenda [emplea el término chador], preceptiva para la mujer según la ortodoxia musulmana, que cubre totalmente el cuerpo y gran parte de la cara" (cursiva mía). Su preferencia por el término chador se refleja en el número de veces que la emplea: nueve en un solo editorial. 
Por su parte, El Mundo, antes de ofrecer su posicionamiento definitivo, condiciona: "Si la palabra chador significa en este caso llevar un pañuelo en la cabeza..." (16 de febrero, cursiva mía). En conjunto, emplea indistintamente los términos chador y pañuelo islámico (siete veces el primero y cinco el segundo).

El País se decanta por emplear hiyab (cinco veces), aunque también emplea velo (en dos ocasiones) y pañuelo (en una). En todo caso, en su presentación habla de una niña que "quiere acudir a la escuela con la cabeza cubierta con el hiyab o pañuelo tradicional en la cultura musulmana" (cursiva mía).

Parece, pues, que los diarios que más se ajustan terminológica y conceptualmente a lo que luego se ha de valorar son El Mundo y El País. No es sólo, pues una cuestión léxica (chador o pañuelo), sino de semántica.

Otro elemento interesante de la presentación del tema tiene también que ver con el estilo léxico que se emplea. Veamos cómo lo hace cada uno de los diarios (la cursiva es mía):

$A B C$ : "UNA niña marroquí de 13 años lleva sin asistir a clase desde octubre porque su padre le obliga a utilizar el chador".

El Mundo: "El caso de la niña marroquí que está sin escolarizar debido a que su padre rechazó enviarla al colegio porque no se le permitía llevar el chador" (16 de febrero).

El País: "El conflicto planteado por las dificultades de escolarización de una niña marroquí de 13 años, que quiere acudir a la escuela con la cabeza cubierta con el hiyab".

Los tres enfatizan que la niña haya dejado de asistir a la escuela, pero de distinta forma: $A B C$ desea recalcar la dimensión temporal (desde octubre, lo que supone casi medio año) y agencial: [ella] lleva sin asistir. Mientras, El Mundo señala simplemente que está sin escolarizar, eligiendo una forma verbal menos activa. Por su parte, El País prefiere una fórmula impersonal y sin verbo al hablar de dificultades de escolarización.

En la segunda parte de la oración se recalca el papel del padre: le obliga a utilizar el chador, en el caso de $A B C$, o rechaza enviarla al colegio porque no se le permitía llevarlo, en el caso de El Mundo. El País no cree relevante señalar este aspecto.

Sólo teniendo en cuenta estas presentaciones, ya se pueden apreciar tres maneras de acercarse al problema radicalmente distintas que crean diferentes predisposiciones en el lector. Tal vez fuese ya posible deducir los puntos de vista respectivos de la forma en que cada uno plantea las premisas.

\section{El velo en la escuela: entre el rechazo frontal y la aceptación con matices}

El único punto en el que los tres diarios convergen sin matices es en la necesidad de garantizar el principio fundamental de la escolarización obligatoria de la niña. A partir de ahí, los puntos de vista varían muy significativamente.

$A B C$ plantea el asunto como una colisión entre valores o principios: "Se trata de un caso de conflicto entre la libertad religiosa y el respeto a principios constitucionales básicos, como el de la dignidad de la persona y la igualdad entre los sexos". No es pues, sólo una expresión religiosa: "Es también una expresión de la discriminación contra la mujer y de la marginación a la que se le pretende someter en 
la vida social y cotidiana". Consecuentemente, $A B C$ se muestra contrario al uso del chador en la escuela, si bien expresa este punto de vista aludiendo a la obligatoriedad de la escolarización. Tras reconocer la "complejidad del caso", afirma no obstante que "está claro que debe prevalecer el respeto a los principios fundamentales en los que se asienta la convivencia y que garantiza la Constitución. La escolarización obligatoria es un deber inexcusable para los padres y para las autoridades académicas. Bajo ningún pretexto puede ser legítima la pretensión del padre de que su hija no acuda a clase si no va vestida con el chador". Al centrarse en esta arista de la escolarización y en la ilegitimidad de que la niña no asista a clase, $A B C$ soslaya que la niña pueda acudir a ella con el hiyab. Pero, en todo caso, no es esquivo en su conclusión: "Tratándose, de un centro público (o, como en este caso, privado concertado), el derecho de las autoridades educativas a imponer la escolarización y a impedir el uso del chador parece evidente".

El Mundo tiene una posición algo titubeante en su primer editorial (día 16), pero se muestra mucho más claro en el segundo (día 18), en el que afirma que no existe ningún conflicto de valores o principios, sino una trasgresión del derecho de la niña a usar la prenda: "La primera trasgresión del derecho de la adolescente a llevar el chador...". Para este diario se trata de un debate complejo que "no puede despacharse con un sí o no al uso del chador" (día 16), cuya resolución ideal pasaría por que el padre permitiera asistir a la hija con el mismo uniforme que el resto de colegiales. Pero, ya que el padre se niega, "las autoridades educativas no pueden de ningún modo castigar a la niña por la falta de sensibilidad y la intolerancia de su padre" (día 16). Vemos cómo la postura de El Mundo no es del todo coherente, pues si llevar el chador es un derecho (transgredido), no se entiende que querer llevarlo suponga una muestra de falta de sensibilidad y un acto de intolerancia...

En todo caso, como hemos visto, considera importante distinguir de qué se habla: considera que si por chador se entiende la túnica que les cubre todo el cuerpo a las mujeres, estaríamos "ante un símbolo de discriminación sexual de las mujeres, y por ello debe ser repudiado en un país en el que la Constitución garantiza la igualdad de sexos" (16 de febrero). Pero a continuación añade: "Si la palabra chador significa en este caso llevar un pañuelo en la cabeza, no debe ser proscrito porque no interfiere el normal desenvolvimiento de la actividad escolar". $Y$ concluye con un poco frecuente ejercicio de autocrítica cultural: "...es mejor optar por la tolerancia, pues nuestra propia cultura continúa plagada de referencias machistas que nadie intenta erradicar a golpe de prohibición".

La postura de El Mundo sería, pues, favorable a la presencia del hiyab en la escuela. En un ejemplo de lo que sería una medida propia de un programa multicultural, este diario afirma: "...es necesario actualizar ahora viejos planteamientos que no salvaguardan los derechos de los alumnos con creencias diferentes a la predominante. Este es el caso de las estrictas normas de uniformidad, que deben flexibilizarse necesariamente para permitir que niñas como Fátima puedan mantener la tradición del chador. Bastaría con admitir el pañuelo islámico como una prenda más aunque fuera, por ejemplo, del mismo color que el resto del uniforme" (día 18, cursiva mía).

Sin embargo, y en otro ejemplo de la ambivalente postura del diario, en otro lugar El Mundo habla, no de la trasgresión del derecho de la niña, sino de la imposición de llevar el pañuelo: "La imposición del velo no deja de ser una expresión simbólica perfectamente asumible, pero que la tolerancia mal entendida no nos obligue a asimilar otras tradiciones incompatibles con los derechos humanos" (4 de marzo). Se entiende, pues, que acceder a la imposición de llevar el velo es un acto de tolerancia. No se trata ya, pues, de un derecho, y el hecho de reconocer su ejercicio no es por tanto un ejercicio de respeto o de reconocimiento debido (en el sentido que Charles Taylor da a este concepto. Véase, por ejemplo, Taylor, 1993) 
Por su parte, El País no cree que exista ningún conflicto entre el aconfesionalismo de la escuela, o los principios constitucionales en general, y la libertad religiosa de la niña. Así, se muestra crítico con la postura del colegio público: "La dirección del colegio público en el que a partir de mañana deberá escolarizarse la niña marroquí ha sustentado la teoría, más que discutible, de que el uso del hiyab es discriminatorio y contradice los valores constitucionales que informan la enseñanza pública en España". Considera, por tanto, que este asunto "debe y puede tener solución en el respeto de tales principios y valores" y cree que la utilización del pañuelo islámico es perfectamente legítima siempre que no implique un acto de proselitismo: "Es decir, sí al velo como expresión individual y respetable de una creencia o costumbre, pero no a su exhibición con intención proselitista, como puede ser el caso cuando su exhibición forma parte de campañas de militancia fundamentalista". El País, por tanto, estaría en contra del velo político, tal y como fue definido en el apartado 3.1.

Al contrario que $A B C$, El País considera que el principio fundamental de la escolarización obligatoria exige que se deba permitir el velo para favorecerla, lo relevante es la escolarización: "El uso del hiyab no atenta contra las normas democráticas que deben ser respetadas por todos. Lo dañino y contrario a la cultura democrática es desescolarizar a las niñas al llegar a la enseñanza secundaria, como sucede a veces en algunos colectivos de inmigrantes".

Así pues, para $A B C$ hay un conflicto de principios en el que deben prevalecer el respeto a los preceptos constitucionales, la igualdad entre los sexos y la obligatoriedad de la escolarización, mientras que para El País no se produciría tal conflicto y el uso del hiyab en la escuela no atentaría contra tales normas. Por su parte, El Mundo mantiene un punto de vista titubeante que ejemplifica la complejidad de las implicaciones que conlleva el asunto del velo. En todo caso, si nos alejamos del cuadro para poder contemplarlo con una perspectiva más amplia, los respectivos puntos de vista devienen más claros. En el fondo, es el Islam lo que está en el punto de mira.

\section{Alejando el zoom: ¿es sólo el velo lo que está en cuestión?}

La importancia que adquiere el debate del velo sólo puede ser entendida si se contempla como parte del debate más amplio acerca de la integración de la población inmigrada de origen arabomusulmán. En otro caso, probablemente no pasaría de ser un asunto menor, carente de trascendencia política y mediática. Concretamente en el caso español, ha de ser visto como una consecuencia del shock al que se enfrentó el mundo tras los atentados terroristas ocurridos en Estados Unidos el 11 de septiembre de 2001 (no olvidemos que el debate del hiyab tuvo lugar a principios de 2002). Estos ataques supusieron un enorme impulso para la ideología del choque de civilizaciones, que había sido elaborada por Samuel P. Huntington a principios de la década de 1990 (véase, por ejemplo, Huntington, 1997) y la supuesta incompatibilidad entre Islam y democracia.

En última instancia, los diarios defienden sus opiniones en este contexto e interpretan el asunto del hiyab como parte de este panorama global, como uno de los escenarios en que se desarrolla la pugna entre los supuestamente irreconciliables universos culturales occidentales y árabomusulmanes. Para los diarios opinar sobre el Islam es opinar sobre el multiculturalismo. Al respecto, el análisis de los editoriales del bienio 2001-2002 revela que la prensa toma dos posturas básicas: por un lado, suscribe la tesis del choque de civilizaciones entre el Islam y Occidente, como $A B C$ y, en menor medida, El Mundo; por otro, se apunta al diálogo de civilizaciones (o culturas), como El País, 
antes de que este concepto tuviera las connotaciones que hoy posee ${ }^{7}$. En cierto modo, estos posicionamientos son coherentes con los puntos de vista que cada diario expresa acerca de la gestión del pluralismo cultural interior.

$A B C$ es tajante al respecto. En su editorial del 26 de octubre de 2002, ilustrativamente titulado "El Islam y Occidente" (la visión dicotómica y polarizada es así llevada al propio título), afirma ya al inicio el carácter indiscutible del choque entre civilizaciones que estaríamos viviendo: "La más torpe manera de encarar la realidad consiste en negarla. Por mucho que algunos se esfuercen en rechazarlo, en los últimos años asistimos a la eclosión de una serie de conflictos que, de un modo u otro, apuntan a un choque entre civilizaciones". Como muestras de dicho conflicto, estarían los atentados del 11 de septiembre, la situación de Oriente Medio, Irak, Bali, el asalto al teatro de Moscú, incluso el francotirador de Washington no quedaría fuera de sospecha. Detrás de todos estos casos "aparece la sombra del fundamentalismo islámico".

El resto del editorial da una visión del Islam claramente negativa: "No se trata de condenar al Islam, ni de negar que no todos sus seguidores son fundamentalistas. Incluso, con cierta benevolencia, puede sobrevolarse sobre muchas expresiones del Corán y pensar que se encuentra en una etapa de su evolución semejante al que pasó hace siglos la civilización cristiana occidental. Aún así, no es posible negar, porque lo declaran sus responsables, que una parte nutrida de la civilización islámica ha declarado la guerra total a Occidente" (cursiva nuestra).

El párrafo precedente supone una auténtica carga de profundidad contra todo atisbo de diálogo o entendimiento entre culturas. El efecto que consigue es la equiparación de hecho entre Islam y fundamentalismo. Y de esta forma se sugiere sutilmente una conexión entre Islam y terrorismo, pues el término fundamentalismo se asocia habitualmente con el de terrorismo (por ejemplo: terrorismo fundamentalista): se trata, pues de una implicación (Van Dijk, 1985: 192-94) con la que el diario puede activar los modelos (Van Dijk, 1997: 45) de sus lectores acerca del Islam, la religión musulmana, el fundamentalismo y los ataques terroristas islamistas.

$A B C$ desea dejar claro, no obstante, que este análisis no debe conducir "ni a la xenofobia, ni a la intolerancia, ni al belicismo", pero afirma que "nuestra civilización se encuentra en peligro y que hay que hacer frente al peligro". Esta actitud no tendría nada que ver ni "con el imperialismo ni con el avasallamiento, sino con la defensa de unos principios que han generado unas formas de civilización y de convivencia superiores a todas las hasta ahora conocidas" ( $A B C, 26$ de octubre de 2002).

Sin embargo, en mi opinión, sí es posible encontrar elementos de etnocentrismo y de de desprecio hacia el Islam. En conjunto, el concepto de islamofobia (Malcom D. Brown, 2000) podría servir para caracterizar algunos de los postulados de $A B C$ (y, en menor medida, de El Mundo). Desde esta perspectiva, el Islam es visto como: 1) inferior a la cultura occidental, irracional, bárbaro, sexista; 2) un bloque monolítico y estático que no responde a los cambios; 3) violento, agresivo, amenazante, defensor del terrorismo y embarcado en un choque de civilizaciones; 4) algo separado, extraño, ajeno, sin valores en común con otras culturas y sin afectar ni ser afectado por éstas.

${ }^{7}$ El 21 de septiembre de 2004, en la 59a Asamblea General de la ONU, el Presidente del Gobierno español Rodríguez Zapatero hizo su propuesta de Alianza de Civilizaciones entre Occidente y el mundo árabomusulmán como medio de luchar contra el terrorismo por vías no militares. Con ello se rescataba del olvido una idea propuesta tres años antes por el presidente iraní Jatami, destinada a desarrollar un Diálogo entre civilizaciones. 
El Mundo comparte en esencia la idea del choque de civilizaciones que mantiene $A B C$ y se hace eco de las opiniones que Oriana Fallaci expresó poco después de los atentados del 11 de septiembre en EE.UU., y que este diario publicó en tres entregas. A través de Fallaci, El Mundo afirmó que existe "una guerra en la que no se combate por el territorio, sino por los valores, por una forma de vida" (EI Mundo, 3 de octubre de 2001); un choque de civilizaciones entre la occidental, tolerante y respetuosa, y la islámica, que se percibe como amenazante: "los valores europeos y norteamericanos permiten la convivencia de gentes de otras culturas, de otras razas, de otras religiones, en su territorio. (...) nosotros respetamos su cultura, pero ellos no respetan la nuestra". Porque el problema, es "el desinterés de una parte de los inmigrantes musulmanes por adaptarse a la forma de vida de los países que los acogen".

Efectivamente, según Fallaci, en esta guerra el enemigo no sería únicamente exterior, sino que estaría asentado dentro. Si bien El Mundo cree que esto es exagerado, la forma en que lo critica no es demasiado clara: "Decir a continuación que «los más aguerridos» guerreros del Islam son los inmigrantes instalados en Europa resulta exagerado. Aunque los 19 suicidas de Nueva York y Washington llevaran un estilo de vida plenamente occidental, no es atribuible a todo musulmán la voluntad de cometer atentados que ellos tenían" (cursiva mía). Esta última negación, tan similar a la de $A B C$ que hemos visto más arriba, es realmente sorprendente: ¿acaso es posible pensar lo contrario, que es atribuible a todo musulmán la voluntad de cometer atentados que ellos tenían?

Sin embargo, y una vez que el diario ha parecido compartir las opiniones de la autora, hasta el punto de confundirse en ocasiones a quién de ambos corresponden, concluye: "Si lo hay o no lo hay [el choque de civilizaciones], no es algo que se vaya a dilucidar de hoy a mañana".

Por su parte, El País se apunta a la vaga idea de un diálogo entre culturas o civilizaciones. Y lo hace a raíz de la visita de Estado a España del presidente de Irán Jatamí, en la que "ha surgido el compromiso mutuo de impulsar el 'diálogo de civilizaciones' con iniciativas concretas que podrían culminar en una reunión de gran altura" (El País, 31 de octubre de 2002). Aparte de defender este proyecto, poco más ofrece el diario en relación con este tema, pues apenas le dedica espacio.

En definitiva, resulta difícil entender el debate del velo si no se tiene en cuenta el debate más amplio actualmente en curso en la mayoría de países occidentales acerca de la integración de la población extranjera (de primera y segunda generación). Más concretamente, ha de ser entendido en un momento histórico crucial, en el que se libra una lucha entre los ruidosos partidarios del choque de civilizaciones (en ambas civilizaciones) y los defensores del diálogo y entendimiento posible entre culturas.

Si bien se puede sostener un punto de vista crítico con el velo desde una perspectiva feminista y emancipadora (en tanto el hiyab es un símbolo de subordinación de las mujeres y constituye una práctica desigualitaria), no es este el caso de la prensa contraria al pañuelo islámico. Desde estos sectores se sugiere, más bien, que tolerar el velo será una concesión irresponsable, un paso en la dirección de la quiebra societaria, de la balcanización social, del intrínsecamente fragmentador multiculturalismo. En el fondo, estos sectores aspiran al ideal del asimilacionismo, esto es, a la propuesta de que "las minorías adopten la lengua, los valores, las normas, las señas de identidad de la cultura dominante y, en paralelo, [vayan] abandonando su cultura propia" (Graciela Malgesini y Carlos Giménez, 2000: 51). Veamos un ejemplo extraído del $A B C$, publicado el 20 de marzo de 2001: "Mientras el [pluralismo] favorece la asimilación y la integración, el [multiculturalismo] conduce a la fragmentación y a la balcanización. El pluralismo contribuye a la libertad y a la tolerancia. El 
multiculturalismo, a la destrucción de los valores liberales y a la intolerancia. La comunicación entre las culturas es factor de enriquecimiento. La fragmentación en guetos culturales conduce a un aislamiento empobrecedor. El inmigrante no tiene derecho a destruir los valores de la sociedad que lo acoge y sí lo tiene a ser admitido sólo cuando la integración sea posible (...)".

En última instancia, es posible identificar a quienes sostiene un punto de vista etnocéntrico basado en la consideración de la cultura occidental como la única garantía de libertad, progreso y democracia, y quienes creen en el diálogo intercultural como vía para el enriquecimiento mutuo de las sociedades. Los primeros parecerían desconocer las palabras de Hans Magnus Enzensberger (1992: 74): "Y en cuanto a los bárbaros, no es necesario que esperemos su llegada; siempre han estado entre nosotros".

\section{Referencias}

Álvarez Dorronsoro, Ignasi (1994). Los retos de la inmigración. En Contreras, Jesús (Comp.), Los retos de la inmigración. Racismo y pluriculturalidad (pp. 25-55). Madrid: Talasa.

Bañón Hernández, Antonio (2002). Discurso e inmigración. Propuestas para el análisis de un debate social. Murcia: Universidad de Murcia.

Bessis, Sophie (2002). Occidente y los otros. Historia de una supremacía. Madrid: Alianza.

Bessis, Sophie y Belhassen, Souhayr (1994). Mujeres del Magreb. Lo que está en juego. Madrid: Horas y horas la editorial.

Botton, Lena de, Puigvert, Lidia y Taleb, Fátima (2004). El velo elegido. Barcelona: El Roure.

Brown, Malcom D. (2000). Conceptualising Racism and Islamophobia. En Ter Wal, Jessika y Verkuyten, Maykel, Comparative Perspectives on Racism (pp. 73-90). Ashgate: Andershot.

Cachón Rodríguez, Lorenzo (2002). La formación de la 'España inmigrante’: mercado y ciudadanía. REIS. Revista Española de Investigaciones Sociológicas, 97, 95-126.

Castles, Stephen y Davidson, Alastair (2000). Citizenship and Migration. Globalization and the politics of belonging. Houndmills: MacMillan.

Cobo Bedia, Rosa (1999). Multiculturalismo, democracia paritaria y participación política. Política y Sociedad, 32, 53-65.

Colectivo IOÉ (1999). Inmigrantes, trabajadores, ciudadanos. Una visión de las migraciones desde España. Valencia: Universitat de Valéncia.

Commission de réflexion sur l'application du principe de laïcité dans le République (2003). Rapport au president de la Republique. Disponible en http://lesrapports.ladocumentationfrancaise.fr/BRP/034000725/0000.pdf (versión española de Andrea Lorca Pérez disponible en http://informestasi.iespana.es).

El Hamel, Chouki (2002). Muslim Diaspora in Western Europe: The Islamic Headscarf (Hijab), the Media and Muslims' Integration in France. Citizenship Studies, 6 (3), 293-308.

Enzensberger, Hans Magnus (1992). La gran migración. Barcelona: Anagrama.

Freedman, Jane (2004). Secularism as a Barrier to Integration? The French Dilemma. International Migration, 42 (3), 5-27.

Hervieu-Léger, Danièle (1999). El pasado en el presente: una nueva definición de la laïcité en la Francia multicultural. En Berger, Peter L. (Ed.), Los límites de la cohesión social. Conflicto y mediación en las sociedades pluralistas (pp.75-134). Barcelona: Galaxia Gutenberg y Círculo de lectores. 
Huntington, Samuel P. (1997). El choque de las civilizaciones y la reconfiguración del orden mundial,. Barcelona: Paidós.

Killian, Caitlin (2003). The Other Side of the Veil: North African Women in France Respond to the Headscarf Affaire. Gender \& Society, 17 (4), 567-590.

Loi n² 2004-228 du 15 mars 2004 encadrant, en application du principe de laïcité, le port de signes ou de tenues manifestant une appartenance religieuse dans les écoles, collèges et lycées publics. [Ley $n^{\circ}$ 2004-228 del 15 de marzo de 2004 que enmarca, en aplicación del principio de laicidad, la tenencia de símbolos o ropa que manifiesten una pertenencia religiosa en los colegios, escuelas y liceos públicos].

Malgesini, Graciela y Giménez, Carlos (2000). Guía de conceptos sobre migraciones, racismo e interculturalidad. Madrid: Los Libros de la Catarata.

Moualhi, Djaouida (2000). Mujeres musulmanas: estereotipos occidentales versus realidad social. Papers: Revista de Sociología, 60, 291-304.

Read, Jen'nan Ghazal y Bartkowski, John P. (2000). To Veil or Not to Veil? A Case Study of Identity Negotiation among Muslim Women Living in Austin, Texas. Gender \& Society, 14 (3), 395417.

Taylor, Charles (1993). El multiculturalismo y "la política del reconocimiento". México D.F.: FCE.

Van Dijk, Teun A. (1985). News Analysis. Case Studies of International and National News in the Press. New Jersey: Lawrence Erlbaum.

Van Dijk, Teun A. (1997). Racismo y análisis crítico de los medios. Barcelona: Paidós.

\section{Historia editorial}

Recibido: $15 / 04 / 2007$

Aceptado: 06/05/2007

\section{Formato de citación}

Abella Vázquez, Carlos M. (2007). La aparición de los discursos sobre el multiculturalismo en España: el debate del velo en la prensa escrita. Athenea Digital, 11, 83-103. Disponible en http://psicologiasocial.uab.es/athenea/index.php/atheneaDigital/article/view/324/327.

Carlos Manuel Abella Vázquez. Doctor en Sociología por la Universidade da Coruña (2006), centro en que el que cursó la Licenciatura (1993-1998), siendo Premio Extraordinario de Licenciatura (1999). Fue becario de investigación (1999), becario predoctoral Xunta de Galicia (2000-2002), investigador contratado (2004-2006), profesional liberal (2003-2006) y actualmente consultor en la empresa privada. 


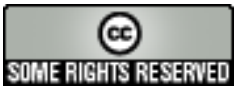

Este texto está protegido por una licencia Creative Commons.

Usted es libre de copiar, distribuir y comunicar públicamente la obra bajo las siguientes condiciones:

Reconocimiento: Debe reconocer y citar al autor original.

No comercial. No puede utilizar esta obra para fines comerciales.

Sin obras derivadas. No se puede alterar, transformar, o generar una obra derivada a partir de esta obra.

Resumen de licencia

$\underline{\text { Texto completo de la licencia }}$ 